Original Article

\title{
A Fundamental Study to Observe Correlation at Molecular Level between Bio-Samples of Patients and Indicated Homoeopathic Medicines
}

\author{
Rajat Chattopadhyay ${ }^{1}$ and C. R. Mahata ${ }^{1,2 *}$ \\ ${ }^{1}$ MBHMC \& Hospital, Howrah- 711104. \\ 2IIEST Shibpur, Howrah-711103. \\ *corresponding author: crmahata@gmail.com
}

\begin{abstract}
Background: 'Like cures like' is the principle that is followed in homoeopathy. Substances which cause disorder in healthy individuals are used as medicines to treat similar patterns of disorder in sick persons. This article aims to find out whether any similarity picture exists at molecular level also that can make a selection of medicines based on scientifically measurable parameters.
\end{abstract}

Method: It consisted of getting frequency domain signatures for the dielectric functions of (i) biofluids (blood serums) of six arthritis patients and (ii) medicines selected for them on the basis of macroscopic similarity. Then, they were compared to find existence/absence of similarity between them.

Results: Medicines administered to the six patients were Rhus tox 200cH, Thuja 30cH, Medorrhinum 200cH. The dielectric loss at resonance for the bio-fluids of the patients and the medicines are given in the body of the article.

Conclusion: It was observed that the patients who had beneficial results with their medicines have a similarity (resonance frequency matching) in spectral signatures of the two groups of substances i.e. bio-fluids (blood serum) of patients and the indicated remedies. This similarity was not evident in the cases where medicines failed to benefit the patients. These results are explorative and enthusiastic, but as the research is limited only to patients with arthritis, generalization could not be drawn. Further work with more rigor is required to establish the facts and apply generalizations to other disease condition. In future, this might be a new tool to integrate the fundamental research with clinical applications in homeopathy.

Keywords: dielectric functions, resonance frequency matching, spectral signatures, Homoeopathic Principle at the molecular level.

\section{Introduction}

The proving symptoms of medicines (in healthy individuals) are matched with manifesting disease symptoms of the patient in the homoeopathic system of medicine. Such type of correlation is not in terms of quantified measurable parameters of physical science. This article aims to find out whether any similarity picture exists at a molecular level that can make a selection of medicines based on scientifically measurable parameters. The immediate question is what should be those parameters, particularly for the ultrahigh diluted (UHD) homeopathic preparations especially beyond $12 \mathrm{c}$ of dilution. Prior to this, we must be clear about the real nature of UHDs. 'Structural concept' may be the most acceptable hypothesis for

OPEN ACCESS

Cite as: Chattopadhyay R, Mahata CR. A Fundamental Study to Observe Correlation at Molecular Level between BioSamples of Patients and Indicated Homoeopathic Medicines. Int J High Dilution Res. 2015;15(3): 11-17. 
explaining medicinal value of UHDs which may be stated as: A substance is to be recognized as a medicine if it has the capability of curing disease(s) while its medicinal property is to be attributed to molecular structure of vehicle like water or of distinct chemical substance when it exists ${ }^{1}$. Following are some of the important studies to support this hypothesis:

'Water polymer' was the terminology used by Barnard $\mathrm{GO}^{2}$ to explain homoeopathy in a theoretical manner. In contrast, Boyd $\mathrm{WE}^{3}$ conducted many successful experiments on the same subject. The lacuna was that explanation for the emanations detected by his 'emanometer', were missing during that period. Much later, a new hypothesis suggested by Chattopadhyay $\mathrm{R}$ et $\mathrm{al}^{4}$ proposed that this phenomenon might have originated from structured vehicle getting excitation from ambient electromagnetic radiation. 'Clathrates' was another terminology used by Anagnostatos GS et $\mathrm{al}^{5}$ in which structural model was called structured water, explaining the formation of clathrates at different stages of serial dilution. Many researchers were attracted to explain exceptional properties of water. Chaplin M enlisted 2388 investigations on water 6 . Another important hypothesis made by Maity $\mathrm{T}$ et al7,8 and Mahata $\mathrm{CR}^{9,10}$ showed experimentally that water structures were present in medicines both below and above the Avogadro limit of 12c. Some experimental results given by Roy Rustum et al ${ }^{11,12}$ also support the structural concept. The experiments made by Davenas $\mathrm{E}$ et $\mathrm{al}^{13}$ on human basophile degranulation showed that 'memory' of the original substance is retained in serial dilution followed by vortexing even beyond the Avogadro limit. It has no direct relation with homoeopathy but its relevance to the dilution/potentisation of homoeopathy is obvious. 'Water memory' may be the popular term, but we prefer to call it 'induced structure'. The hydrogen-bonded network of water remained modified even in dilutions beyond Avogadro limit was shown by Rey Louis $^{14}$ in his investigation with a dilution of substance like $\mathrm{LiCl}$ and $\mathrm{NaCl}$. It may be assumed that such modification will lead to variation in the structure of molecular clusters of the vehicle. Even ultra-diluted (1012) DNA and RNA solutions continuing to emit electromagnetic radiations like the undiluted substances as reported by Montagnier $\mathrm{L}$ et $\mathrm{al}^{15}$ are explainable in terms of structures 4 . Elia V et al 16 also supported the concept of a structural model for extremely diluted substances. But, Mahata $\mathrm{CR}^{1}$ was possibly the first person to advance it as a generalized concept.

\section{Method}

'Structural concept' receives stronger credence by the investigations of Mahata $\mathrm{CR}^{9,10}$ using dielectric dispersion techniques. It is based on the principle of resonance in acoustical (or, longitudinal) mode of vibration of macromolecules subjected to alternating electric field. Around such a resonance, electrical property of the material exhibits abrupt changes - changes of dielectric loss and permittivity with anomalous dielectric dispersion. The peak value of dielectric loss occurs at resonance frequency 7,8 So, in frequency domain measurements monitoring of dielectric loss will detect the frequency of resonance and monitoring of permittivity will confirm the occurrence of anomalous dielectric dispersion. A simple rule relating fundamental resonance frequency and length of structure (equal to half-wavelength) is:

(Frequency) $\mathrm{x}$ (Wavelength) $=($ Velocity of sound in the medium $)$

This says that resonance frequency is related to the dimension of structure. ${ }^{8}$ Therefore, a change of resonance frequency will imply a change of dimension of the macromolecule.

\section{OPEN 2 ACCESS}

Cite as: Chattopadhyay R, Mahata CR. A Fundamental Study to Observe Correlation at Molecular Level between BioSamples of Patients and Indicated Homoeopathic Medicines. Int J High Dilution Res. 2015;15(3): 11-17. 
This is the guiding principle adopted here to show that even with same chemical composition (namely, $\mathrm{H}_{2} \mathrm{O}$ ) different homeopathic medicines may have different resonance frequencies and be structurally different.7, 8,9,10

This study inspired us to inquire whether the concept like macroscopic matching (that is, the symptom similarity) can be extended to molecular level! By using the same technique can we find any correlation at the molecular level between patients' bio-samples and probable medicines? We selected medicines for several cases of arthritis which were the indicated ones in each case. Then, the frequency domain signatures for dielectric functions of these medicines and blood serum of the patients were recorded with experimental set up as shown in Figure I.

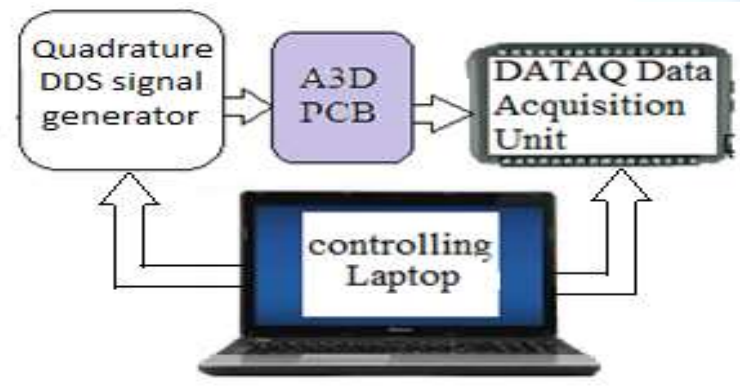

Fig - I: Block diagram of experimental setup.

The Quadrature (Direct Digital Synthesizer) DDS using AD9854 IC chip was manufactured in Shenzhen, China; the Data Acquisition Unit was DI-149 manufactured by WINDAQ, USA. We have indigenously made the A3D PCB (Printed Circuit Board) giving outputs representing real and imaginary component values of the dielectric function. Its functional diagram is shown in Figure II.

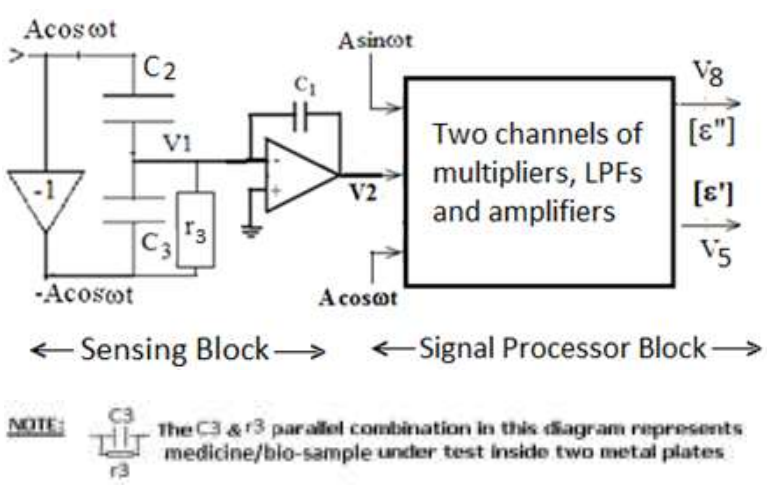

Fig - II: Functional diagram of the A3D PCB

Here, $C_{1}$ is an air capacitor. $C_{2}$ is the capacitance of a reference cell formed by two metal plates measuring 3"x 4" (76.2 mm x $101.6 \mathrm{~mm}$ ). The two metal plates were separated by $1.5 \mathrm{~mm}$ with a polyethene pouch inserted therein, which was serving as its dielectric material. The capacitance was $300 \mathrm{pF}$. C3 in parallel with R3 represents an imperfect capacitor which was also formed by two metal plates as for $\mathrm{C} 2$, and its dielectric material comprised of the test material, which were globules made of sugar (six in number of size no.10) soaked in medicine or bio-sample of subject (as the case may be) and then dried and finally dissolved in $5 \mathrm{ml}$ of water (procured from M/s Hahnemann Publishing Co, Kolkata; free from all chemical and mechanical impurities) through syringe in a polythene pouch which was to be placed between the metal plates. Its capacitance was also adjusted to $300 \mathrm{pF}$ by fine-adjustment of the gap between the metal plates. The frequency domain signature was taken from $1.0 \mathrm{MHz}$ to $30.0 \mathrm{MHz}$. Adjustments were made for the frequency step, ramp rate of the Quadrature DDS signal generator and sampling rate of Data Acquisition unit to get a graphical record of 1 div equal to $1 \mathrm{MHz}$ along the $\mathrm{x}$-axis and $0.25 \mathrm{~V} / \mathrm{div}$ along the $\mathrm{y}$-axis in the graphs of Fig. III to Fig. VIII representing dielectric loss (imaginary part of a dielectric function) as a function of frequency.

\section{Results}

\section{OPEN ACCESS}

Cite as: Chattopadhyay R, Mahata CR. A Fundamental Study to Observe Correlation at Molecular Level between BioSamples of Patients and Indicated Homoeopathic Medicines. Int J High Dilution Res. 2015;15(3): 11-17. 
In all the spectra of Bio-fluids and medicines given in Fig. III to Fig. VIII the standard deviation (SD) was within +/- 1\%.

Patient -1: female, 50yrs, OPD registration no. H-F47249. Symptoms: pain in left knee relief from continued motion and heat, pain aggravated during first motion, the red triangular tip of the tongue, predominant sycotic past and family history. Diagnosis osteoarthritis. Medicine prescribed - Rhus tox $200 \mathrm{cH}$. The resonance frequency of bio-fluid of patient - 22.8 $\mathrm{MHz}$ and that of applied medicine - $22.8 \mathrm{MHz}$. Status - Marked improvement.

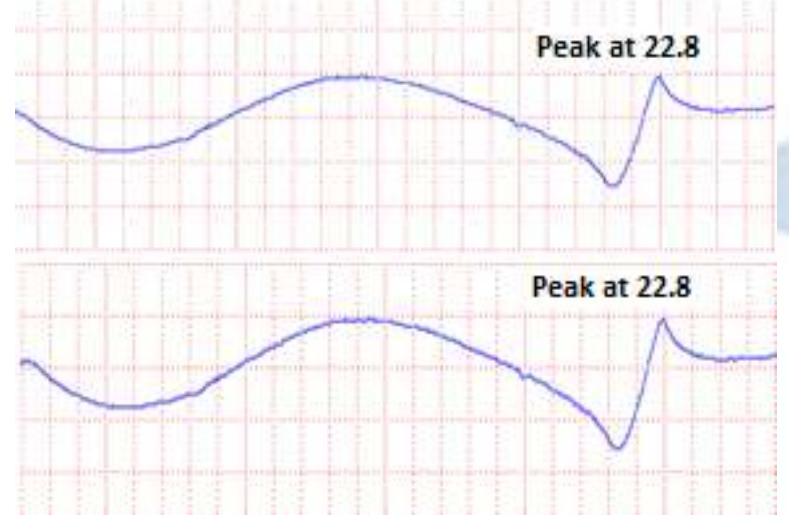

Fig III(a) Spectrum for Bio fluid of Patient 1 Fig. III(b) Spectrum for Rhus tox $200 \mathrm{cH}$

Patient - 2: Female, 40 yrs, OPD registration no. H-F47248. Symptoms: pain in both knees relief from motion, being occupied physically and heat, pain aggravated during rest, the light reddish tip of the tongue, sycotic constitution with generalities. Diagnosis osteoarthritis. Medicine prescribed - Rhus tox $200 \mathrm{cH}$. The resonance frequency of bio-fluid of patient - 23.6 MHz and that of applied medicine - 22.8 MHz. Status - Mild improvement.

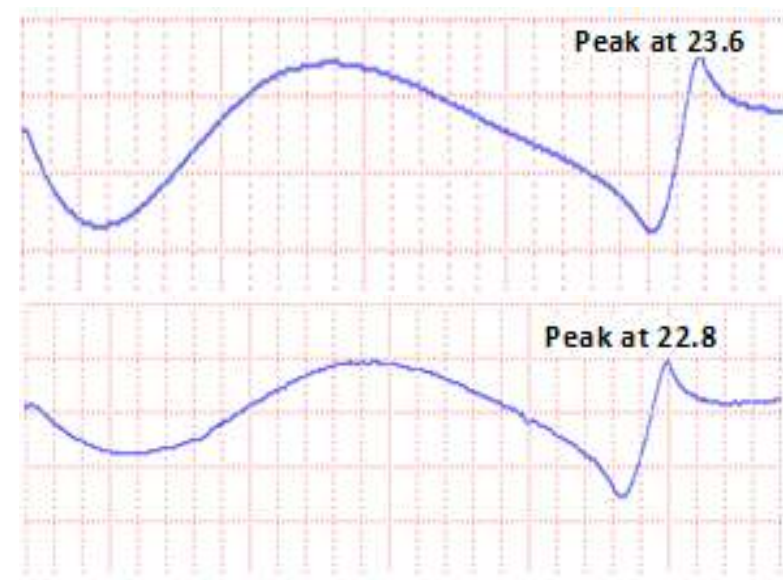

Fig IV(a) Spectrum for Bio fluid of Patient 2 Fig. IV(b) Spectrum for Rhus tox $200 \mathrm{cH}$

Patient - 3: Female, 62 yrs, OPD registration no. H-F99308. Symptoms: pain in both knees with occasional numbness of the extremities relief from hot fomentation only, pain aggravated during flexing, pedunculated warts, multiple small black warts on face and neck area, sycotic constitution with generalities, the fearful dream of a dead person and falling from a height, chilly patient. Diagnosis - osteoarthritis. Medicine prescribed - Thuja $30 \mathrm{cH}$. The resonance frequency of bio-fluid of patient $-23.6 \mathrm{MHz}$ and that of applied medicine - $23.6 \mathrm{MHz}$. Status - improvement continues.

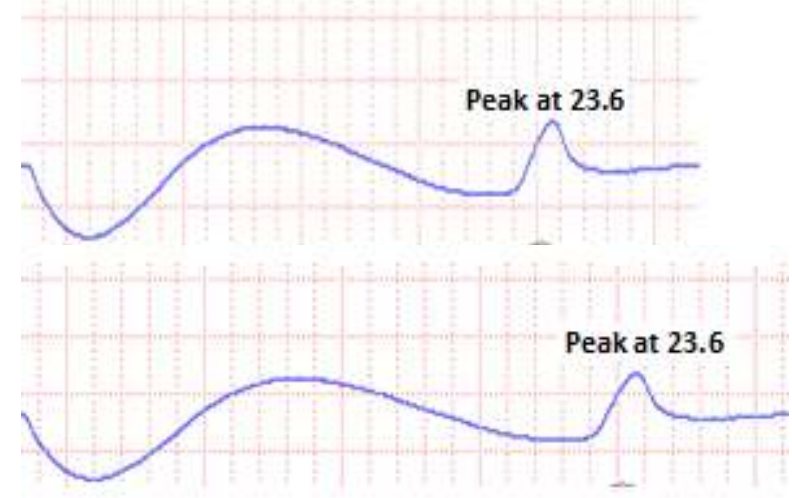

Fig V(a) Spectrum for Bio fluid of Patient 3 Fig. V(b) Spectrum for Thuja 30cH

\section{OPEN ACCESS}

Cite as: Chattopadhyay R, Mahata CR. A Fundamental Study to Observe Correlation at Molecular Level between BioSamples of Patients and Indicated Homoeopathic Medicines. Int J High Dilution Res. 2015;15(3): 11-17. 
Patient - 4: Female, 54yrs, OPD registration no. H-F 96336. Symptoms: pain in middle fingers of both upper extremities with some swelling, recurrent red eye, sycotic constitution with generalities, the frequent dream of dead parents, chilly patient, all RA markers negative, ESR high. Diagnosis seronegative Rheumatoid arthritis. Medicine prescribed - Thuja 30cH. The resonance frequency of bio-fluid of patient $-24.2 \mathrm{MHz}$ and that of applied medicine $-23.6 \mathrm{MHz}$. Status - No significant improvement till date related to pain but patient feels better (in relation to systemic review) and still under treatment.

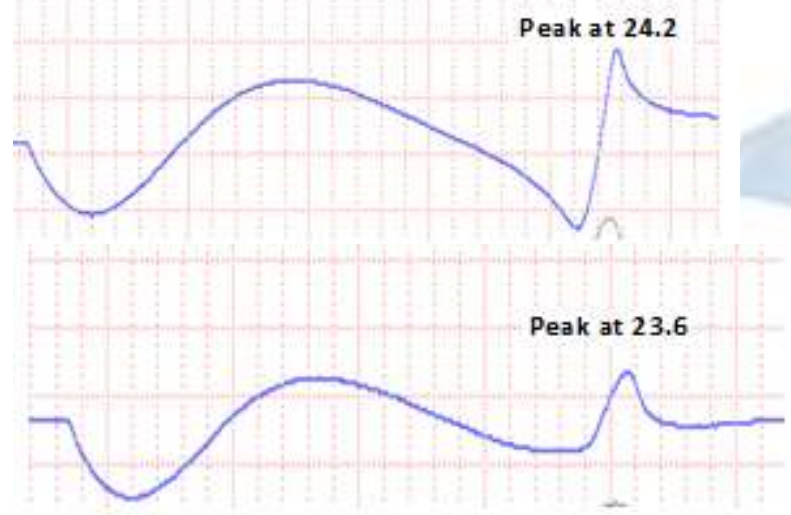

Fig. VI(a) Spectrum for Bio fluid of Patient 4 Fig. VI(b) Spectrum for Thuja $30 \mathrm{cH}$

Patient - 5: Female, 48 yrs, OPD registration no. H-F91122. Symptoms: pain in back and both knees, aggravated during walking and amelioration after application of heat, numbness of lower extremities and sensation of falling down during walking, flabby constitution with strong sycotic generalities, dream of falling from height, chilly patient, papilloma under tongue, very small whitishbrownish spots all over the skin surface, osteophytes around knee joint with degenerative changes in X-rays. Diagnosis Osteoarthritis. Medicine prescribed - Thuja $30 \mathrm{cH}$. The resonance frequency of bio-fluid of patient - 23.4 MHz and that of applied medicine - 23.6 MHz. Status -Improved.

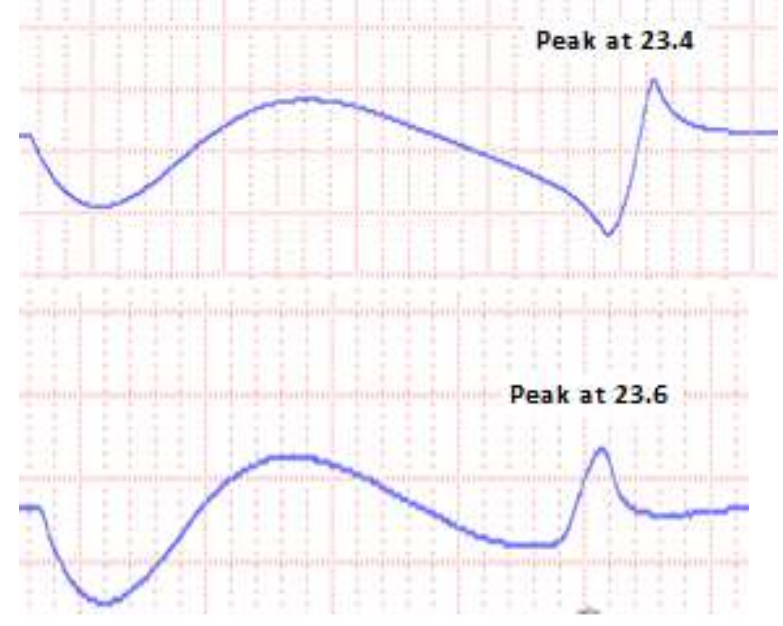

Fig. VII(a) Spectrum for Bio fluid of Patient 5 Fig. VII(b) Spectrum for Thuja 30cH

Patient - 6: Female, 32 yrs, OPD registration no. H-F067134. Symptoms: pain \& soreness of both knees \& ankle joints, more pain felt in knee joints, aggravated at noon, new moon and amelioration after application of heat, open air \& flexing the affected joint, short height with stout fleshy constitution with strong sycotic generalities, poor memory, ambithermal patient, auto-immune markers negative, degenerative changes are seen in $\mathrm{x}$ rays. Diagnosis - Osteoarthritis. Medicine prescribed - Medorrhinum 200cH. The resonance frequency of bio-fluid of patient $22.8 \mathrm{MHz}$ and that of applied medicine - 28.6 MHz. Status -No improvement.

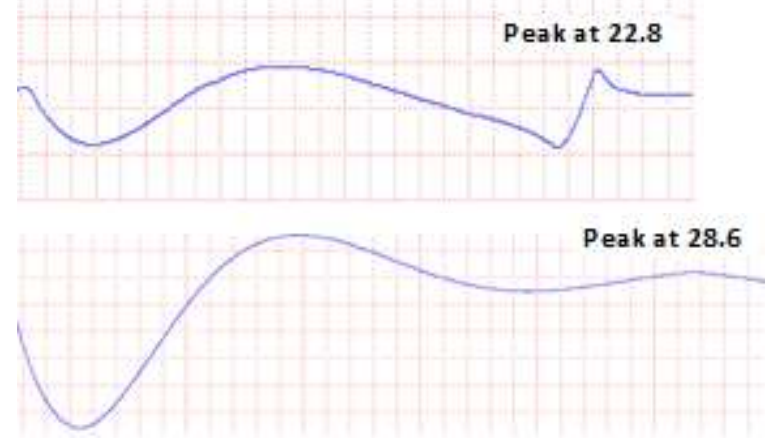

\section{OPEN $(2)$ ACCESS}

Cite as: Chattopadhyay R, Mahata CR. A Fundamental Study to Observe Correlation at Molecular Level between BioSamples of Patients and Indicated Homoeopathic Medicines. Int J High Dilution Res. 2015;15(3): 11-17. 
Fig. VIII(a) Spectrum for Bio fluid of Patient 6

Fig. VIII(b) Spectrum for Medorrhinum 200cH

The resonance frequencies of bio-samples and possible homoeo-medicines are compared through the Table below.

\section{References}

1. Mahata CR. The structural model explains high potency homoeopathic medicines and leads to a generalized understanding of medicines. Intl. J. CAM 2015; 1(2); 1-3.

2. Barnard GO. Microdose paradox - a new concept. J. American Inst. of Homeopathy, July-Aug 1965; 205-212.

3. Sir Thomas Horder's communication to the Royal Society of Medicine published as "The electronic reactions of Abrams". The British Medical Journal, Jan24 1924; 179-185.

4. Chattopadhyay R, Sadhukhan M, Pal A, Sutradhar A, Syam P, Mahata CR. On electromagnetic signals from vastly diluted DNA solutions, potentised medicines and even metals. Wesleyan Journal of Research, Oct 2012; 5(1): 50-55.

5. Anagnostatos GS, Vithoulkas G, Garzonis P, Tavouxoglou C. A working hypothesis for homoeopathic microdiluted remedies. The Berlin Journal of Research in Homoeopathy June 1991; 1(3): 141-147.

6. Chaplin M. Website: www1.lsbu.ac.uk/water/water_struct ure_science.html. (Last accessed on 18 Aug 2015.)

7. Maity Tanmoy, Ghosh D, Mahata CR. Theory and Instrumentation related to Potentised Homoeopathic Medicines. IE(I) Journal - ID 2007; 88: $27-31$.

8. Maity T, Ghosh D, Mahata CR. Effect of Dielectric Dispersion on Potentised Homoeo-Medicines. Homeopathy 2010; 99(2): 99-103. applications in homeopathy.

\section{OPEN ACCESS}

Cite as: Chattopadhyay R, Mahata CR. A Fundamental Study to Observe Correlation at Molecular Level between BioSamples of Patients and Indicated Homoeopathic Medicines. Int J High Dilution Res. 2015;15(3): 11-17. 
9. Mahata CR. Dielectric Dispersion Studies Indicate Change in Structure of Water by Potentised Homeopathic Medicines. IE(I) 2013; 93(4): 231-35.

10. Mahata CR. Dielectric Dispersion Studies of Some Potentised Homoeopathic Medicines Reveal Structured Vehicle. Homeopathy 2013; 102(4): 262-267.

11. Roy Rustum, Tiller WA, Bell Iris, Hoover MR. The structure of liquid water; novel insights from materials research; potential relevance to homeopathy. Materials Research Innovations Online Sept 2005; pp.577 $-608$.

12. Rao Manju Lata, Roy Rustum, Bell Iris $\mathrm{R}, \mathrm{H}$ Richard. The defining role of structure (including epitaxy) in the plausibility of homeopathy. Homeopathy 2007; 96 (3): 175-182.

13. Davenas E, Beauvais F, Amara J, etal. Human basophil degranulation triggered by very dilute antiserum against IgE. Nature 30 Jun 1988; 333: 816-18.

14. Rey L. Thermo-luminescence of ultrahigh dilutions of lithium chloride and sodium chloride. Physica- A 2003; 323: 67-74.

15. Montagnier L, Aäissa J, Ferris S, Montagnier JL, Lavallee C. Electromagnetic signals are produced by aqueous nanostructures derived from bacterial DNA sequences. Interdiscip Sci Comput Life Sci 2009; 1 $-10$.

16. Elia V, Ausanio G, Gentile F, Germano R, Napoli E, Niccoli M. Experimental evidence of stable water nanostructures in extremely dilute solutions, at standard pressure and temperature. Homeopathy 2014; 103 (4): pp 44-50.

Received: Apr 21, 2016. Accepted: Sep 23, 2016.

(C) International Journal of High Dilution Research.

Not for commercial purposes.

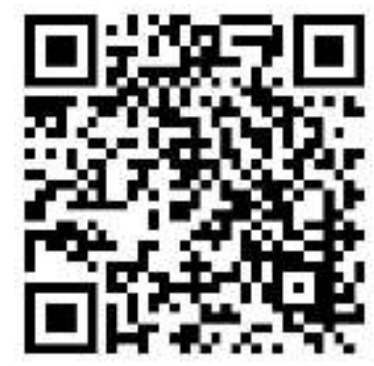

OPEN (?) ACCESS

Cite as: Chattopadhyay R, Mahata CR. A Fundamental Study to Observe Correlation at Molecular Level between BioSamples of Patients and Indicated Homoeopathic Medicines. Int J High Dilution Res. 2015;15(3): 11-17. 